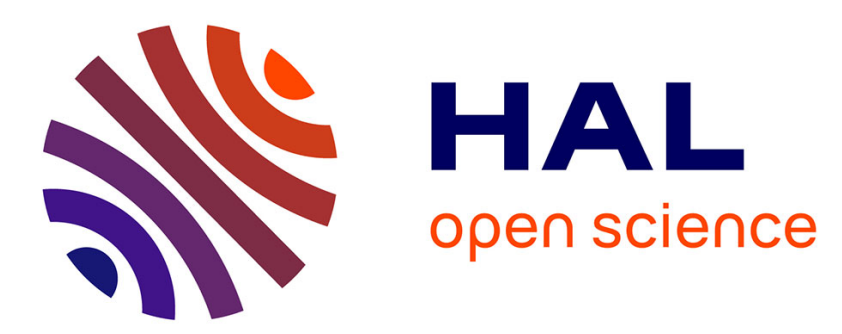

\title{
Hyperbolic Delaunay Complexes and Voronoi Diagrams Made Practical
}

Mikhail Bogdanov, Olivier Devillers, Monique Teillaud

\section{To cite this version:}

Mikhail Bogdanov, Olivier Devillers, Monique Teillaud. Hyperbolic Delaunay Complexes and Voronoi Diagrams Made Practical. Proceedings of the 29th Annual Symposium on Computational Geometry, Jun 2013, Rio, Brazil. pp.67-76, 10.1145/2462356.2462365 . hal-00833760

\section{HAL Id: hal-00833760 https://hal.inria.fr/hal-00833760}

Submitted on 13 Jun 2013

HAL is a multi-disciplinary open access archive for the deposit and dissemination of scientific research documents, whether they are published or not. The documents may come from teaching and research institutions in France or abroad, or from public or private research centers.
L'archive ouverte pluridisciplinaire HAL, est destinée au dépôt et à la diffusion de documents scientifiques de niveau recherche, publiés ou non, émanant des établissements d'enseignement et de recherche français ou étrangers, des laboratoires publics ou privés. 


\title{
Hyperbolic Delaunay Complexes and Voronoi Diagrams Made Practical
}

\author{
Mikhail Bogdanov Olivier Devillers Monique Teillaud \\ INRIA Sophia Antipolis - Méditerranée, http://www.inria.fr/sophia/geometrica/
}

\begin{abstract}
We study Delaunay complexes and Voronoi diagrams in the Poincaré ball, a conformal model of the hyperbolic space, in any dimension. We elaborate on our earlier work on the space of spheres [18], giving a detailed description of algorithms. We also study algebraic and arithmetic issues, observing that only rational computations are needed. All proofs are based on geometric reasoning, they do not resort to any use of the analytic formula of the hyperbolic distance. This allows for an exact and efficient implementation in 2D. All degenerate cases are handled. The implementation will be submitted to the CGAL editorial board for future integration into the CGAL library.
\end{abstract}

\section{INTRODUCTION}

As D. Eppstein states: "Hyperbolic viewpoint may help even for Euclidean problems" [23]. ${ }^{1}$ He gives two examples: the computation of 3D Delaunay complexes of sets lying in two planes [8], and optimal Möbius transformation and conformal mesh generation [5]. Hyperbolic geometry is also used in applications like graph drawing [29, 24].

Several years ago, we showed that the hyperbolic Delaunay complex and Voronoi diagram can easily be deduced from their Euclidean counterparts [18, 8]. As far as we know, this was the first time when the computation of hyperbolic Delaunay complexes and Voronoi diagrams was addressed. Since then, the topic appeared again in many publications. Onishi and Takayama write [31, p. 4]: "The algorithm and the theorem were already given in [18]. Here, we could naturally rediscover their algorithm...", their proofs only relying on algebraic computations instead of geometric reasoning. Nielsen and Nock transform the computation of the Voronoi diagram in the non-conformal Klein model to the computation of a Euclidean power diagram [30]. Many other references can be found in [34] (which does not mention [18, 8], though).

${ }^{1}$ see also http://www.ics.uci.edu/ eppstein/pubs/geom-hyperbolic.html
None of the above papers shows interest in practical aspects, especially algebraic and arithmetic aspects, which are well known to be crucial for exactness and efficiency of implementations. Still, implementations are necessary for applications in various fields, such as neuro mathematics [15].

In this paper, we stick to the Poincaré ball model of the hyperbolic space, which is conformal, i.e., preserves hyperbolic angles. Due to this property, the model is used in a wide range of applications (see for instance [28, 41, 27]). We elaborate on our preliminary work [18, 8], giving a detailed description of algorithms allowing to compute the hyperbolic Delaunay complex and Voronoi diagram in any dimension. All degenerate cases are handled. All proofs rely on purely geometric proofs, avoiding any computation and any use of the hyperbolic distance formulas. We show that only simple arithmetic computations on rational numbers are needed. The algorithm was implemented in $2 \mathrm{D}$ in an exact and efficient way. The implementation will soon be submitted to the CGAL editorial board for future integration into the CGAL library.

We first recall some background on the space of spheres (Section 2), Euclidean Voronoi diagrams and Delaunay triangulations. Section 3 recalls basics on hyperbolic geometry, and their interpretation in the space of spheres. In Section 4, we study hyperbolic Voronoi diagrams and Delaunay complexes, and we present algorithms. Section 5 shows, using geometric reasoning, that the computation and embedding of hyperbolic Delaunay complexes and Voronoi diagrams only use rational computations, but for Voronoi vertices whose coordinates are algebraic numbers of degree two. Section 6 presents the implementation, it gives precisions on algebraic and arithmetic aspects, and presents experimental results, in dimension 2 .

\section{THE SPACE OF SPHERES}

$\mathbb{E}^{d}$ denotes the $d$-dimensional Euclidean space, $<., .>$ the scalar product, and \|.\| the Euclidean norm.

The space of spheres gives a correspondence between spheres of $\mathbb{E}^{d}$ and points of $\mathbb{E}^{d+1}$ [4, Chapter 20] [18]. As stated in Proposition 1, it yields a different way of seeing the well-known duality between Voronoi diagrams and arrangements [22].

Let $\chi$ denote the last coordinate in the space of spheres $\mathbb{E}^{d+1}$. The direction of the $\chi$-axis is called vertical.

A Euclidean sphere $S$ centered at $c$ with radius $r$ is denoted as $S=(c, r)$ and has equation $S(x)=0$ in $\mathbb{E}^{d}$, where:

$$
S(x)=\|x\|^{2}-2<c, x>+\|c\|^{2}-r^{2} .
$$



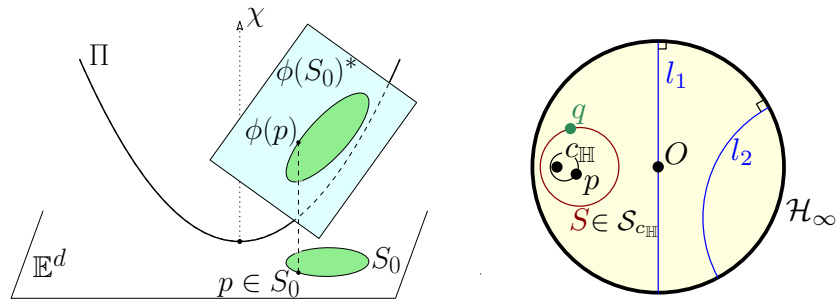

Figure 1: Left: The space of spheres.

Right: The Poincaré disk model of $\mathbb{H}^{2}$ : hyperbolic lines $l_{1}, l_{2}$, hyperbolic circle $S$ with center $c_{\mathbb{H}}$.

The map $\phi$ associates $S$ to the point

$$
\phi(S)=(c, \chi) \in \mathbb{E}^{d+1}, \quad \chi=\|c\|^{2}-r^{2} .
$$

We can embed $\mathbb{E}^{d}$ into $\mathbb{E}^{d+1}$ by identifying it with the hyperplane $\chi=0$. By the embedding, $\phi(S) \in \mathbb{E}^{d+1}$ projects vertically on $\mathbb{E}^{d}$ to the center $c$ of $S$. The points of $\mathbb{E}^{d}$, considered as spheres of null radius, map by $\phi$ to the unit paraboloid of $\mathbb{E}^{d+1} \Pi: \chi=\|c\|^{2}$. Spheres of $\mathbb{E}^{d}$ map to points below $\Pi$, whereas a point above $\Pi$ corresponds to an imaginary sphere, i.e., a sphere whose radius is an imaginary complex number.

The pencil of spheres determined by two spheres $S_{1}$ and $S_{2}$ is the set of spheres whose equations are the affine combinations of the equations of $S_{1}$ and $S_{2}$ :

$$
S: \quad S(x)=\alpha \cdot S_{1}(x)+(1-\alpha) \cdot S_{2}(x), \quad \alpha \in \mathbb{E} .
$$

This pencil is mapped by $\phi$ to the line through $\phi\left(S_{1}\right)$ and $\phi\left(S_{2}\right)$ in the space of spheres.

The set of spheres of $\mathbb{E}^{d}$ orthogonal to a given sphere $S_{0}$ is represented in $\mathbb{E}^{d+1}$ by the polar hyperplane $\phi\left(S_{0}\right)^{*}$ of point $\phi\left(S_{0}\right)=\left(c_{0}, \chi_{0}\right)$ with respect to $\Pi$. The equation of this polar hyperplane is obtained by polarizing the equation of $\Pi$ in $S_{0}$ (see Figure 1-left):

$$
\phi\left(S_{0}\right)^{*}: \frac{\chi+\chi_{0}}{2}=<c_{0}, c>.
$$

In particular, a point (seen as a sphere of null radius) and a sphere are orthogonal if and only if the point lies on the sphere. The intersection of $\phi\left(S_{0}\right)^{*}$ and $\Pi$ is the image by $\phi$ of the set of points of $\mathbb{E}^{d}$ lying on $S_{0}$, i.e., $\phi\left(S_{0}\right)^{*} \cap \Pi$ vertically projects on $\mathbb{E}^{d}$ to $S_{0}$. For a point $p \in \mathbb{E}^{d}$, the set of spheres passing through $p$ maps to the hyperplane $\phi(p)^{*}$ tangent to $\Pi$ at point $\phi(p) \in \Pi$.

The lower half space of $\mathbb{E}^{d+1}$ limited by $\phi(p)^{*}$ (i.e., the half space which does not contain $\Pi$ ) represents the spheres of $\mathbb{E}^{d}$ that enclose $p$ in their interior open ball. In a symmetric way, the upper halfspace, denoted as $\Phi(p)$ represents the spheres that do not enclose $p$ in their interior.

In general, for a flat $A$ in $\mathbb{E}^{d+1}$, we denote as $A^{*}$ the polar of $A$ with respect to $\Pi$ :

$A^{*}=\left\{(x, \chi) \in \mathbb{E}^{d+1} \mid \forall a=\left(x_{a}, \chi_{a}\right) \in A, \frac{\chi+\chi_{a}}{2}=<x_{a}, x>\right\}$,

If $A \oplus B$ denotes the affine sum of two flats $A$ and $B$, standard algebra shows that

$$
(A \cap B)^{*}=A^{*} \oplus B^{*} .
$$

\section{Euclidean Voronoi diagram and Delaunay triangulation}

Let $\mathcal{P}$ be a finite set of points in $\mathbb{E}^{d}$. For the sake of simplicity, we assume points to be in non-degenerate position. This is in fact not a restriction of our method, since we can always come down to this situation by using a symbolic perturbation scheme as in [21] (the paper describes the 3D case but the scheme is general).

The Euclidean Voronoi diagram $\operatorname{VD}_{\mathbb{E}}(\mathcal{P})$ of $\mathcal{P}$ is the partition of $\mathbb{E}^{d}$ into Voronoi cells $V_{\mathbb{E}}\left(p_{i}\right)=\left\{x \in \mathbb{E}^{d} \mid \forall p_{j} \in \mathcal{P}\right.$, $\|$ $\left.x-p_{i}\|\leq\| x-p_{j} \|\right\}$. The Euclidean Delaunay triangulation $\operatorname{DT}_{\mathbb{E}}(\mathcal{P})$ is the geometric dual of the Voronoi diagram. For further reading on these extensively studied data structures, see for instance $[16,9,1,2]$.

Each cell $V_{\mathbb{E}}\left(p_{i}\right)$ of the Voronoi diagram can be interpreted as the set of centers of spheres passing through $p_{i}$ and enclosing no point of $\mathcal{P}$. The set of empty spheres, i.e., spheres that do not enclose any point of $\mathcal{P}$, is mapped by $\phi$ in the space of spheres to the intersection of the upper half spaces $\Phi(p)$ of $\mathbb{E}^{d+1}, p \in \mathcal{P}$, as defined above. The boundary of this intersection is a convex polyhedron $U_{\mathcal{P}}$, whose facets are tangent to $\Pi$.

Proposition 1 ([18]). The Voronoi diagram $V D_{\mathbb{E}}(\mathcal{P})$ is the cell complex of dimension d in $\mathbb{E}^{d}$ obtained by vertically projecting the polyhedron $U_{\mathcal{P}}$ onto $\mathbb{E}^{d}$.

If $\sigma$ is a $k$-simplex of $\mathrm{DT}_{\mathbb{E}}(\mathcal{P})$, we denote as $\mathcal{P}_{\sigma}$ the set of its vertices. The dual of $\sigma$ is a $(d-k)$-face of $\operatorname{VD}_{\mathbb{E}}(\mathcal{P})$, which is the vertical projection of a $(d-k)$-face $u_{\sigma}$ of $U_{\mathcal{P}}$. Any point in $u_{\sigma}$ is the image by $\phi$ of the center of a sphere passing through the vertices of $\sigma$. Thus, $u_{\sigma}$ is a convex polyhedron included in the $(d-k)$-flat $\bigcap_{p \in \mathcal{P}_{\sigma}} \phi(p)^{*}$ that is the intersection of the hyperplanes dual to $\phi(p)$ for all vertices $p$ of $\sigma$.

The $k$-simplex $\sigma$ is incident to $m(k+1)$-simplices $\tau_{i}$, $0 \leq i<m$, of $\operatorname{DT}_{\mathbb{E}}(\mathcal{P})$. The set of vertices of a simplex $\tau_{i}$ of this family is $\mathcal{P}_{\tau_{i}}=\mathcal{P}_{\sigma} \cup\left\{p_{i}\right\}$, for some point $p_{i} \in \mathcal{P} \backslash \mathcal{P}_{\sigma}$. The duals of these simplices $\tau_{i}$ determine the boundary of $u_{\sigma}$ on the $(d-k)$-flat $\bigcap_{p \in \mathcal{P}_{\sigma}} \phi(p)^{*}$. To summarize:

$$
u_{\sigma}=\bigcap_{p \in \mathcal{P}_{\sigma}} \phi(p)^{*} \bigcap \bigcap_{0 \leq i<m} \Phi\left(p_{i}\right)
$$

\section{THE POINCARÉ BALL MODEL OF THE HYPERBOLIC SPACE}

The hyperbolic space $\mathbb{H}^{d}$ [4, Chapter 19] [35, 38] can be represented by several widely used models. There are transformations between these models, as recalled in [11, 36].

In the Poincaré ball model, the $d$-dimensional hyperbolic space $\mathbb{H}^{d}$ is represented as the open unit ball $\mathcal{B}=\left\{x \in \mathbb{E}^{d}\right.$ : $\|x\|<1\}$. The points on the boundary of $\mathcal{B}$ are the points at infinity. The set of such points is $\mathcal{H}_{\infty}=\left\{x \in \mathbb{E}^{d}:\|x\|=1\right\}$. Hyperbolic lines, or geodesics, are represented either as arcs of Euclidean circles orthogonal to $\mathcal{H}_{\infty}$ or as diameters of $\mathcal{B}$. See Figure 1-right for an illustration in 2D. The Poincaré ball model of $\mathbb{H}^{d}$ can be embedded into $\mathbb{E}^{d+1}$ by identifying it with the open unit $d$-disk $\chi=0,\|x\|<1$.

In the space of spheres, the set of points at infinity $\mathcal{H}_{\infty}$ (i.e., the unit sphere in $\mathbb{E}^{d}$ ) is mapped to the point $\phi\left(\mathcal{H}_{\infty}\right)=$ $(0, \ldots, 0,-1)$. Its polar hyperplane is $\pi_{\infty}=\phi\left(\mathcal{H}_{\infty}\right)^{*}: \chi=$ 1 . $\mathbb{H}^{d}$ is mapped to the part of the paraboloid $\Pi$ that lies 


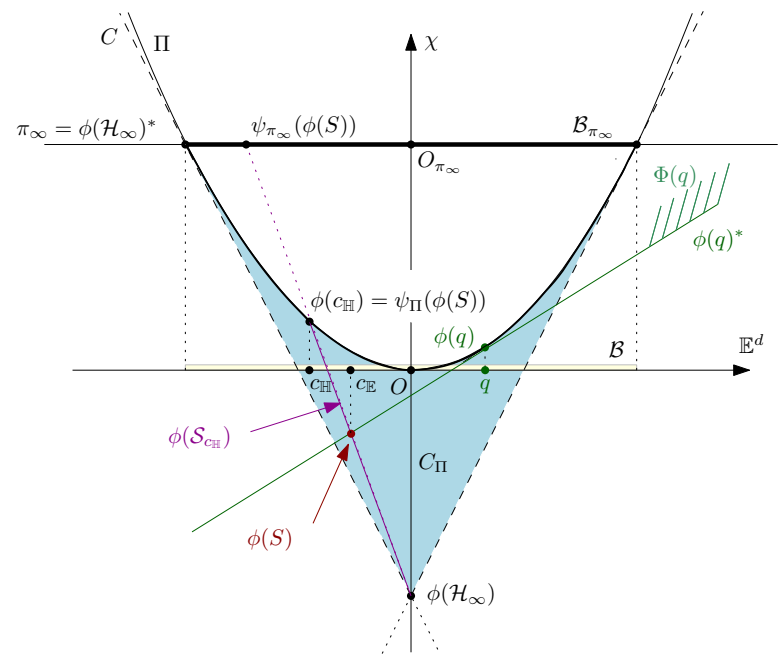

Figure 2: The Poincaré ball model of $\mathbb{H}^{d}$ in the space of spheres. $q \in S, c_{\mathbb{E}}$ is the Euclidean center of $S, c_{\mathbb{H}}$ is its hyperbolic center.

below $\pi_{\infty}$. For a point $x \in \mathcal{H}_{\infty}$, the hyperplane $\phi(x)^{*}$ is tangent to $\Pi$ and passes through $\phi\left(\mathcal{H}_{\infty}\right)$.

Hyperbolic spheres are Euclidean spheres contained in $\mathcal{B}$, but the center of a hyperbolic sphere usually does not coincide with its Euclidean center. Let us consider the pencil of spheres determined by $\mathcal{H}_{\infty}$ and a point $c_{\mathbb{H}} \in \mathcal{B}$ considered as a sphere of radius 0 . Those spheres of the pencil that are contained in $\mathcal{B}$ are the hyperbolic spheres centered at $c_{\mathbb{H}}$. We denote them by $\mathcal{S}_{c_{\mathbb{H}}}$. They represent a collection of nested spheres growing from $c_{\mathbb{H}}$ to $\mathcal{B}$. In the space of spheres $\mathbb{E}^{d+1}, \phi\left(\mathcal{S}_{c \mathbb{H}}\right)$ is the half-open line segment $\left[\phi\left(c_{\mathbb{H}}\right), \phi\left(\mathcal{H}_{\infty}\right)\right)$.

A point $p$ is closer than point $q$ to point $c_{\mathbb{H}}$ for the hyperbolic distance if the sphere of $\mathcal{S}_{c_{\mathbb{H}}}$ that passes through $p$ is inside the sphere of $\mathcal{S}_{c_{H}}$ that passes through $q$. Note that we do not need to consider the explicit expression of the hyperbolic distance. See [3] for its more complete description.

The intersection of the upper half spaces of $\mathbb{E}^{d+1}$ limited by the hyperplanes $\phi(x)^{*}, x \in \mathcal{H}_{\infty}$ forms the cone $C$ with apex $\phi\left(\mathcal{H}_{\infty}\right)$ tangent to $\Pi$ (see Figure 2). $C$ represents the set of Euclidean spheres that do not intersect $\mathcal{H}_{\infty}$. The set of hyperbolic spheres is the set of Euclidean spheres inside $\mathcal{B}$. In the space of spheres, it is mapped to the open subset $C_{\Pi}$ of $C$ that lies below $\pi_{\infty}$ and below $\Pi$. $C_{\Pi}$ can also be seen as $\cup_{x \in \mathcal{B}} \phi\left(\mathcal{S}_{x}\right)$.

Let $S \subset \mathcal{B}$ a hyperbolic sphere mapped to $\phi(S) \in C_{\Pi}$ in the space of spheres. We denote as $\psi_{\Pi}$ the central projection onto $\Pi$ centered at $\phi\left(\mathcal{H}_{\infty}\right): \psi_{\Pi}(\phi(S))$ is the intersection of line $\left(\phi\left(\mathcal{H}_{\infty}\right) \phi(S)\right)$ with $\Pi$. This intersection point is in fact the projection by $\phi$ on $\Pi$ of the hyperbolic center $c_{\mathbb{H}}$ of $S$. We denote as $\psi_{\pi_{\infty}}$ the central projection centered at $\phi\left(\mathcal{H}_{\infty}\right)$ onto $\pi_{\infty}$.

\section{COMPUTING THE HYPERBOLIC DELAUNAY COMPLEX}

Let $\mathcal{P}$ be a finite set of points in $\mathbb{H}^{d}$, represented in the Poincaré ball model.

The hyperbolic Voronoi diagram $\operatorname{VD}_{\mathbb{H}}(\mathcal{P})$ is defined as its Euclidean counterparts, replacing the Euclidean distance by the hyperbolic distance.

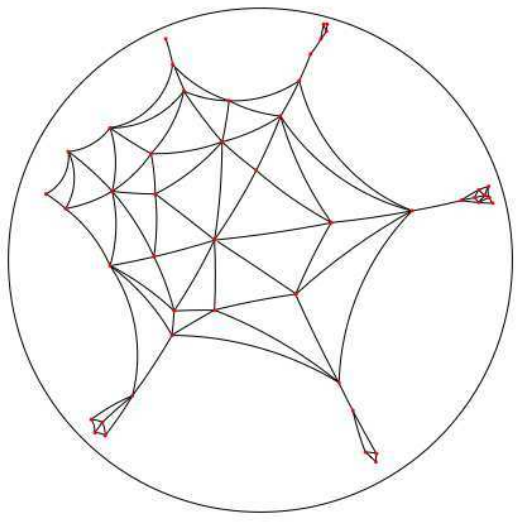

Figure 3: Hyperbolic Delaunay complex.

Let us take a point $x$ in the cell $V_{\mathbb{H}}\left(p_{i}\right)$ of $p_{i}$ in $\operatorname{VD}_{\mathbb{H}}(\mathcal{P})$. $x$ is closer to $p_{i}$ than to any other point in $\mathcal{P}$ for the hyperbolic distance. As already noted, we can avoid considering the hyperbolic distance explicitly, and express this proximity property in other words: a sphere of the pencil generated by the sphere of null radius $x$ and $\mathcal{H}_{\infty}$, and growing from $x$ to the sphere $\mathcal{H}_{\infty}$, meets $p_{i}$ before meeting any other point of $\mathcal{P}$. This illustrates the fact that for every point $x$ in $V_{\mathbb{H}}\left(p_{i}\right)$ there exists a unique sphere $S$ hyperbolically centered at $x$ passing through $p_{i}$ and enclosing no point of $\mathcal{P}$.

A simplex $\sigma$ is an element of the hyperbolic Delaunay complex $\mathrm{DT}_{\mathbb{H}}(\mathcal{P})$ iff there exists a point $c \in \mathbb{H}^{d}$ that is the hyperbolic center of a hyperbolic sphere (i.e., a Euclidean sphere contained in $\mathcal{B}$ ) passing through the vertices of $\sigma$ and enclosing no point of $\mathcal{P}$ in its open interior ball. An example, drawn with our implementation (see Section 6), is shown on Figure 3.

Note that some $k$-simplices in the hyperbolic Delaunay complex may have no $(k+1)$-simplices in the complex incident to them (this is the case of a few edges in Figure 3). This is the reason why we don't call $\mathrm{DT}_{\mathbb{H}}(\mathcal{P})$ a triangulation. $\mathrm{DT}_{\mathbb{H}}(\mathcal{P})$ is still a simplicial complex.

Let us prove a property of $\mathrm{DT}_{\mathbb{H}}(\mathcal{P})$, which will not be used in the algorithm, but which is still interesting in itself.

Proposition 2. The Delaunay complex $D T_{\mathbb{H}}(\mathcal{P})$ is connected.

Proof. For any partition of $\mathcal{P}$ into two sets of points, the next lemma proves that there is an edge of the hyperbolic Delaunay complex between these two sets, which proves the proposition.

Lemma 3. Let $\mathcal{P}$ be partitioned into a set $R$ of red points and a set $B$ of blue points. Then there exist two points $p_{r} \in$ $R$ and $p_{b} \in B$, and an empty sphere $S_{p q}$ passing through $p_{r}$ and $p_{b}$ that is contained in $\mathcal{B}$.

Proof. Let us first construct a sphere $S_{0}$ centered at the origin $O$ by starting from the unit ball $\mathcal{B}$ and reducing its radius until it contains points of only one color. Without loss of generality, $S_{0}$ contains only blue points. Let $p_{r}$ be the red point through which $S_{0}$ passes.

Then we construct another sphere $S_{p q}$, by starting from $S_{0}$ and reducing the radius, while keeping the sphere in the pencil of spheres through $p_{r}$ and tangent to $S_{0}$, until it is empty. Let $p_{b}$ be the blue point through which $S_{p q}$ passes.

We have constructed a sphere $S_{p q}$ whose open interior 
ball is empty, that passes through $p_{r}$ and $p_{b}$, and that is contained in $\mathcal{B}$.

The correspondence between $\operatorname{VD}_{\mathbb{H}}(\mathcal{P})$ and $\operatorname{VD}_{\mathbb{E}}(\mathcal{P})$ can be seen as follows, using the central projection $\psi_{\Pi}$ (see Section 3). Let $f_{\mathbb{E}}$ be a $k$-face of $\operatorname{VD}_{\mathbb{E}}(\mathcal{P})$, i.e., the set of Euclidean centers of empty spheres passing through a subset $\mathcal{P}_{f}$ of $d+1-k$ points of $\mathcal{P}$. By Proposition $1, f_{\mathbb{E}}$ is the vertical projection of a $k$-face $f_{U}$ of $U_{\mathcal{P}}$. Let $c_{\mathbb{E}}$ be a point of $f_{\mathbb{E}}$, i.e., the Euclidean center of an empty sphere $S \subset \mathbb{E}^{d}$ passing through $\mathcal{P}_{f}$. If $S$ is contained in $\mathcal{B}$, the point obtained by centrally projecting $\phi(S) \in f_{U}$ to $\Pi$ from $\phi\left(\mathcal{H}_{\infty}\right)$ is $\psi_{\Pi}(\phi(S))$, which projects vertically onto the hyperbolic center $c_{\mathbb{H}}$ of $S$. This can be summarized as:

Proposition 4 ([18]). The hyperbolic Voronoi diagram $V D_{\mathbb{H}}(\mathcal{P})$ can be obtained by centrally projecting from $\phi\left(\mathcal{H}_{\infty}\right)$ the part of the polyhedron $U_{\mathcal{P}}$ lying in $C_{\Pi}$ to the paraboloid $\Pi$, and projecting the result vertically onto $\mathbb{E}^{d}$.

As a consequence, $D T_{\mathbb{H}}(\mathcal{P})$ is a subcomplex of $D T_{\mathbb{E}}(\mathcal{P})$. It consists of faces of $D T_{\mathbb{E}}(\mathcal{P})$ that admit at least one empty ball passing through its vertices and included in $\mathcal{B}$.

The computation of $\mathrm{DT}_{\mathbb{H}}(\mathcal{P})$ thus consists of the following two steps:

- Compute the Euclidean Delaunay triangulation $\mathrm{DT}_{\mathbb{E}}(\mathcal{P})$

- Extract from $\mathrm{DT}_{\mathbb{E}}(\mathcal{P})$ the simplices that also belong to $\operatorname{DT}_{\mathbb{H}}(\mathcal{P})$.

The rest of this section is devoted to showing how this extraction step can be performed. We first describe variants of this general scheme. The predicate that tests whether a given simplex of $\mathrm{DT}_{\mathbb{E}}(\mathcal{P})$ is also in $\mathrm{DT}_{\mathbb{H}}(\mathcal{P})$ is denoted as is_hyperbolic and will be detailed in Section 4.2.

\subsection{Extracting $\mathbf{D T}_{\mathbb{H}}(\mathcal{P})$ from $\mathbf{D T}_{\mathbb{E}}(\mathcal{P})$ : algo- rithms}

We give several variants of the extraction scheme. The basic one closely follows what was just presented. The second is an improvement that allows to test a smaller number of simplices. Both are static, i.e., they first compute the whole Euclidean triangulation before performing the extraction. The third one (omitted in this abstract, see [6]) is dynamic: it allows to add a point and to update the hyperbolic Delaunay complex while the Euclidean Delaunay triangulation is updated.

\subsubsection{Basic algorithm}

Let us first remark that, if a simplex of $\operatorname{DT}_{\mathbb{E}}(\mathcal{P})$ also belongs to the hyperbolic Delaunay complex $\mathrm{DT}_{\mathbb{H}}(\mathcal{P})$, then all its faces also belong to $\mathrm{DT}_{\mathbb{H}}(\mathcal{P})$. Extracting $\mathrm{DT}_{\mathbb{H}}(\mathcal{P})$ from $\mathrm{DT}_{\mathbb{E}}(\mathcal{P})$ can be done by examining simplices by decreasing dimensions, starting from $d$-simplices. The extraction consists in simply marking each $k$-simplex of $\mathrm{DT}_{\mathbb{E}}(\mathcal{P})$, $k=1, \ldots, d$ as "hyperbolic" or "non-hyperbolic".

For each dimension $k$, we maintain a dictionary $D_{k}$ of simplices to be examined. The dictionary $D_{d}$ initially contains all $d$-simplices of $\mathrm{DT}_{\mathbb{E}}(\mathcal{P})$, other dictionaries are empty. The algorithm proceeds by decreasing dimensions, starting at $k=d$.

For each $k, d \geq k \geq 1$;

while $D_{k} \neq \bar{\emptyset}$, pop first $k$-simplex $\sigma$ of $\mathrm{DT}_{\mathbb{E}}(\mathcal{P})$ out of $D_{k}$; If it is marked already, don't do anything;

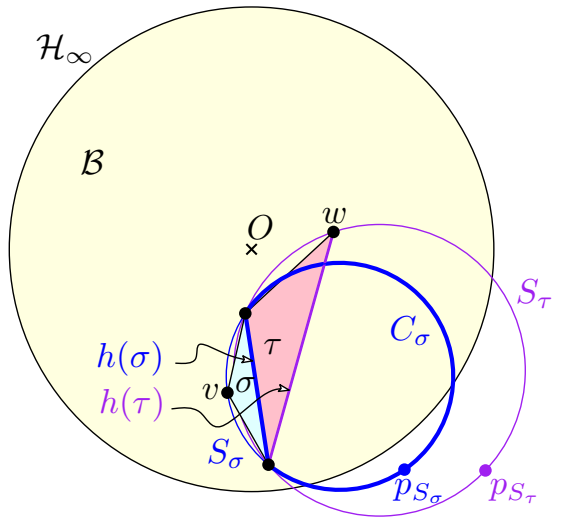

Figure 4: Proof of Prop 6.

If not marked yet, test whether it is a simplex of $\mathrm{DT}_{\mathbb{H}}(\mathcal{P})$; If yes, we mark $\sigma$ as "hyperbolic", as well as all its faces of all dimensions $i$ from $k-1$ down to 1 ;

If not, we mark $\sigma$ as "non-hyperbolic"; its $(k-1)$-faces are inserted into $D_{k-1}$;

end while;

end for.

Anticipating on Section 4.2, in which it will be clear that predicate is_hyperbolic can be evaluated with constant complexity, we get:

Proposition 5. The hyperbolic Delaunay complex of $n$ points in the Poincare ball model $\mathbb{H}^{d}$ can be computed in time $\Theta\left(n \log n+n^{\lceil d / 2\rceil}\right)$.

\subsubsection{Improved extraction scheme}

This improved scheme does not reduce the theoretical complexity given in Proposition 5, but it does reduce the total amount of work. In fact, we could write the running time with respect to the number of simplices that are not in the result that is the size of $\mathrm{DT}_{\mathbb{E}}(\mathcal{P}) \backslash \mathrm{DT}_{\mathbb{H}}(\mathcal{P})$.

We consider that the Euclidean space $\mathbb{E}^{d}$ is compactified to a topological sphere by the addition of a point at infinity. This point at infinity can be linked to all simplices of the convex hull of $\mathcal{P}$. After adding these infinite simplices to it, the Euclidean triangulation $\operatorname{DT}_{\mathbb{E}}(\mathcal{P})$ becomes a triangulation of a combinatorial sphere.

Proposition 6. The graph $G$ whose nodes are the d-simplices of $D T_{\mathbb{E}}(\mathcal{P}) \backslash D T_{\mathbb{H}}(\mathcal{P})$ and the infinite d-simplices of $D T_{\mathbb{E}}(\mathcal{P})$, and whose arcs are adjacency relations through non-hyperbolic facets in $D T_{\mathbb{E}}(\mathcal{P})$, is connected.

Proof. We first remark that the infinite simplices of $\operatorname{DT}_{\mathbb{E}}(\mathcal{P})$ form the set of all simplices that are adjacent to the infinite vertex, so, their graph is connected.

Let $\sigma$ be a finite $d$-simplex of $\operatorname{DT}_{\mathbb{E}}(\mathcal{P})$ that is not in $\mathrm{DT}_{\mathbb{H}}(\mathcal{P})$. The sphere $S_{\sigma}$ circumscribing $\sigma$ intersects $\mathcal{H}_{\infty}$. More precisely, among the $d$ spherical caps on $S_{\sigma}$ that are limited by the supporting (Euclidean) hyperplanes of the facets of $\sigma$ and that do not contain any vertex of $\sigma$, at least one cap intersects $\mathcal{H}_{\infty}$. If there are several such caps, we choose one that contains the point $p_{S_{\sigma}}$ of $S_{\sigma}$ that is the farthest to $O$.

Let us call $C_{\sigma}$ such a cap and $h(\sigma)$ the corresponding facet of $\sigma$ (see Figure 4). Any sphere $S^{\prime}$ passing through 


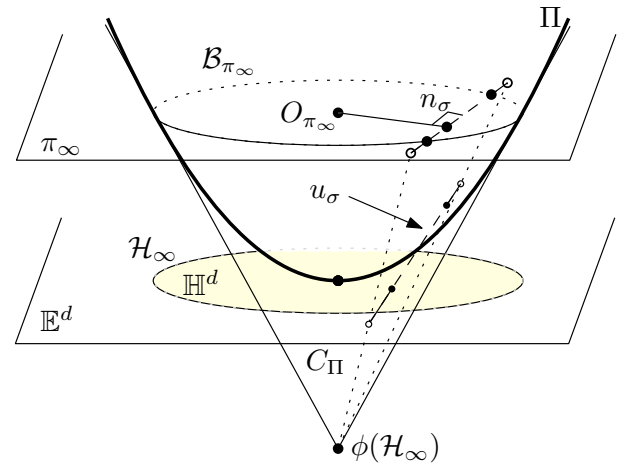

Figure 5: Condition for a simplex $\sigma$ of $\operatorname{DT}_{\mathbb{E}}(\mathcal{P})$ to be a simplex of $\mathbf{D T}_{\mathbb{H}}(\mathcal{P})$.

the vertices of $h(\sigma)$ either encloses the vertex $v$ of $\sigma$ opposite to $h(\sigma)$, or intersects $\mathcal{H}_{\infty}$. Thus, the $(d-1)$-simplex $h(\sigma)$ does not belong to $\mathrm{DT}_{\mathbb{H}}(\mathcal{P})$. Moreover, when $S^{\prime}$ intersects $\mathcal{H}_{\infty}$, it encloses $p_{S_{\sigma}}$, and its point $p_{S^{\prime}}$ farthest to $O$ is such that $\left\|O p_{S^{\prime}}\right\|>\left\|O p_{S_{\sigma}}\right\|$.

Let $\tau$ be the neighbor of $\sigma$ through $h(\sigma)$ in $\mathrm{DT}_{\mathbb{E}}(\mathcal{P})$ and $w$ the vertex of $\tau$ that is not in $h(\sigma)$. Observe that $\tau \notin$ $\mathrm{DT}_{\mathbb{H}}(\mathcal{P})$; otherwise $h(\sigma)$ would have been in $\mathrm{DT}_{\mathbb{H}}(\mathcal{P})$ since $h(\sigma)$ is a face of $\tau$. Then, observe that $\left\|O p_{S_{\tau}}\right\|>\left\|O p_{S_{\sigma}}\right\|$. A path of $d$-simplices can be constructed in graph $G$, using adjacency relations, starting at $\sigma$ and ending at an infinite simplex, by choosing at each step the adjacent simplex through $h(\sigma)$. This path traverses only simplices of $\mathrm{DT}_{\mathbb{E}}(\mathcal{P}) \backslash \mathrm{DT}_{\mathbb{H}}(\mathcal{P})$.

Corollary 7. If $d=2$, there is a bijection $h$ between non-hyperbolic triangles and non-hyperbolic edges.

Proof. In dimension 2, the definition of $h(\sigma)$ appearing in the proof of Proposition 6 does not involve any choice of a good spherical cap, since in two dimensions the three caps are disjoint and only one intersects $\mathcal{H}_{\infty}$. The mapping $h$ has been proved to be injective. It remains to prove that any edge $e \in \mathrm{DT}_{\mathbb{E}}(\mathcal{P}) \backslash \mathrm{DT}_{\mathbb{H}}(\mathcal{P})$ is the image by $h$ of a triangle $\sigma$. Let $S$ be the boundary of an empty ball circumscribing $e$. $S$ intersects $\mathcal{H}_{\infty}$, otherwise $e \in \mathrm{DT}_{\mathbb{H}}(\mathcal{P})$. Then we consider circles of the pencil of circles passing through the vertices of $e$, starting from $S$, and going in the direction that decreases the intersection with $\mathcal{H}_{\infty}$, until we find a vertex $v$ of $\mathcal{P}$. Vertex $v$ and edge $e$ form the Euclidean Delaunay triangle $\sigma$ such that $h(\sigma)=f$.

The improved version of the extraction algorithm consists in "digging" $\mathrm{DT}_{\mathbb{E}}(\mathcal{P})$ : its starts from the infinite $d$-simplices of $\operatorname{DT}_{\mathbb{E}}(\mathcal{P})$, and recursively traverses $G$ using adjacency relations. The recursive traversal stops digging as soon as it can only reach $d$-simplices on which is_hyperbolic is true. During the traversal, faces of all $d$-simplices of $G$ are tested against is_hyperbolic and marked accordingly as in the basic algorithm.

\subsection{Extracting $\mathrm{DT}_{\mathbb{H}}(\mathcal{P})$ from $\mathrm{DT}_{\mathbb{E}}(\mathcal{P})$ : predi- cate}

Let us now explain the test is_hyperbolic, which checks whether a simplex of $\mathrm{DT}_{\mathbb{E}}(\mathcal{P})$ is in $\mathrm{DT}_{\mathbb{H}}(\mathcal{P})$. Let observe right now that this predicate has no degenerate case: as mentioned in Section 3, hyperbolic spheres are Euclidean

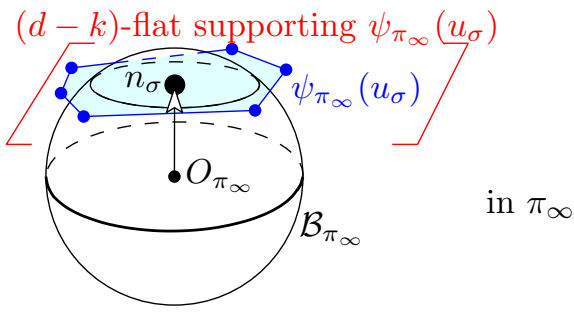

Figure 6: The central projection of $u_{\sigma}$ on $\pi_{\infty}$ intersects the open unit $d$-ball $\mathcal{B}_{\pi_{\infty}}$ in $\pi_{\infty}$.

spheres contained in the open ball $\mathcal{B}$. The only candidate for a degenerate case would be the limit case when the only empty sphere passing through the vertices of a given simplex is tangent to $\partial \mathcal{B}=\mathcal{H}_{\infty}$. Then the simplex is just not hyperbolic, and the case is in fact not degenerate.

We first look at $d$-simplices. Let $\sigma$ be a $d$-simplex of $\mathrm{DT}_{\mathbb{E}}(\mathcal{P})$, dual to a Voronoi vertex, vertically projected from the vertex $u_{\sigma}$ of $U_{\mathcal{P}}$ in $\mathbb{E}^{d+1}$. $u_{\sigma}$ is the image by $\phi$ of the (empty) sphere circumscribing $\sigma$. From Section $3, \sigma$ is a $d$-simplex of $\mathrm{DT}_{\mathbb{H}}(\mathcal{P})$ iff $u_{\sigma}$ lies in $C_{\Pi}$. Since $\mathcal{P} \subset \mathcal{B}$, the circumscribing sphere of $\sigma$ cannot completely lie outside $\mathcal{B}$, so, this equivalence can be rewritten as: $\sigma$ is a $d$-simplex of $\mathrm{DT}_{\mathbb{H}}(\mathcal{P})$ iff $u_{\sigma}$ lies in $C$.

For a general dimension $k \leq d$, the discussion at the end of Section 3 straightforwardly shows that the following conditions are equivalent (see Figure 5):

1. the $k$-simplex $\sigma \in \mathrm{DT}_{\mathbb{E}}(\mathcal{P})$ is a simplex of $\mathrm{DT}_{\mathbb{H}}(\mathcal{P})$

2. $u_{\sigma}$ intersects $C$

3. $\psi_{\Pi}\left(u_{\sigma}\right) \neq \emptyset$

4. $\psi_{\pi_{\infty}}\left(u_{\sigma}\right)$ intersects the open unit $d$-ball $\mathcal{B}_{\pi_{\infty}}$ of $\pi_{\infty}$.

If $\sigma$ is a $k$-simplex of $\operatorname{DT}_{\mathbb{H}}(\mathcal{P})$ whose incident $(k+1)$ simplices do not belong to $\mathrm{DT}_{\mathbb{H}}(\mathcal{P})$, the central projection $\psi_{\pi_{\infty}}\left(u_{\sigma}\right)$ of $u_{\sigma}$ onto $\pi_{\infty}$ is a convex $(d-k)$-polyhedron whose facets lie outside the unit $d$-ball $\mathcal{B}_{\pi_{\infty}}$ in $\pi_{\infty}$ (see Figure 6). The polyhedron $\psi_{\pi_{\infty}}\left(u_{\sigma}\right)$ intersects $\mathcal{B}_{\pi_{\infty}}$, but its boundary does not intersect $\mathcal{B}_{\pi_{\infty}}$, so, the whole intersection of the supporting flat of $\psi_{\pi_{\infty}}\left(u_{\sigma}\right)$ with $\mathcal{B}_{\pi_{\infty}}$ is contained in $\psi_{\pi_{\infty}}\left(u_{\sigma}\right)$. So, it is sufficient to test any well chosen point in the intersection of the supporting flat and the ball $\mathcal{B}_{\pi_{\infty}}$. In hyperplane $\pi_{\infty}$, let $n_{\sigma}$ be the point of the supporting $(d-k)$-flat of $\psi_{\pi_{\infty}}\left(u_{\sigma}\right)$ that is the nearest to the origin $O_{\pi_{\infty}}$ of $\mathcal{B}_{\pi_{\infty}}$. Predicate is_hyperbolic will check whether $\psi_{\pi_{\infty}}\left(u_{\sigma}\right)$ intersects $\mathcal{B}_{\pi_{\infty}}$, i.e., is_hyperbolic $(\sigma)$ is true, iff $n_{\sigma}$ is contained in both the convex polyhedron $\psi_{\pi_{\infty}}\left(u_{\sigma}\right)$ and the ball $\mathcal{B}_{\pi_{\infty}}$.

\section{GEOMETRIC PROOFS FOR ALGE- BRAIC ASPECTS}

In this section we first recall how the Euclidean Delaunay triangulation $\mathrm{DT}_{\mathbb{E}}(\mathcal{P})$ can be computed only using rational computations. Then we show in Section 5.2 that extracting the hyperbolic Delaunay complex $\mathrm{DT}_{\mathbb{H}}(\mathcal{P})$ from $\mathrm{DT}_{\mathbb{E}}(\mathcal{P})$ can also be performed only using rational computations. In Sections 5.3 and 5.4, we consider the geometric embeddings of $\mathrm{DT}_{\mathbb{H}}(\mathcal{P})$ and $\mathrm{VD}_{\mathbb{H}}(\mathcal{P})$ in the Poincaré ball model.

We assume that the coordinates $\left(x_{0}, \ldots, x_{d-1}\right)$ of each point $x$ of $\mathcal{P}$ are rational. 


\subsection{Computing $\mathbf{D T}_{\mathbb{E}}(\mathcal{P})$}

This section quickly recalls basic facts, we refer the reader to the mentioned literature for more details. Many standard algorithms have been proposed to compute $\mathrm{DT}_{\mathbb{E}}(\mathcal{P})$. The CGAL library [14] offers an efficient implementation in $2 \mathrm{D}$ and $3 \mathrm{D}$, based on the incremental construction first proposed by Bowyer [10] and Watson [37]. Several options are proposed for point location, using either some walking strategy [20] or a hierarchical data structure [17].

The robustness of the implementation against arithmetic issues is ensured by following the exact geometric paradigm [39]. The computation of the combinatorial structure underlying $\operatorname{DT}_{\mathbb{E}}(\mathcal{P})$ only relies on the evaluation of two predicates.

The predicate orientation $\left(p_{0}, p_{1}, \ldots, p_{d}\right)$ decides the orientation of $d+1$ points, and boils down to the sign of the determinant of $\left(p_{i_{0}}, p_{i_{1}}, \ldots, p_{i_{d-1}}, 1\right)$ in $\mathbb{E}^{d+1}$.

The predicate $i n \_s p h e r e\left(p_{0}, p_{1}, . ., p_{d}, q\right)$ decides whether $q$ lies in the open interior ball of the sphere passing through the $d+1$ first points. If we assume that these $d+1$ first points are positively oriented, then the predicate is given by the sign of the determinant of $\left(p_{i_{0}}, p_{i_{1}}, \ldots, p_{i_{d-1}},\left\|p_{i}\right\|^{2}, 1\right)$ in $\mathbb{E}^{d+2}$, which is in fact exactly the orientation predicate of $\left(\phi\left(p_{0}\right), \phi\left(p_{1}\right), \ldots, \phi\left(p_{d}\right), \phi(q)\right)$ in the space of spheres.

Only rational operations are needed to evaluate these signs of polynomial expressions. The computation is made both exact and fast by using filtered exact computations [19].

\subsection{Extracting $\mathbf{D T}_{\mathbb{H}}(\mathcal{P})$ from $\mathbf{D} \mathbf{T}_{\mathbb{E}}(\mathcal{P})$}

LEMMA 8. Each rational point of $\mathbb{E}^{d+1}$ is projected by $\psi_{\pi_{\infty}}$ on $\pi_{\infty}$ to a rational point.

Proof. A point $(c, \chi) \in \mathbb{E}^{d+1}$ is projected to $\pi_{\infty}$ following the line through $\phi\left(\mathcal{H}_{\infty}\right)$ to point $(x, 1), x \in \mathbb{E}^{d}$, such that $x=\frac{2}{1+\chi} \cdot c$. Note that $1+\chi$ is the power product of the unit sphere $\mathcal{H}_{\infty}$ and the sphere $S$ such that $\phi(S)=(c, \chi)$.

Lemma 9. The sphere $S$ circumscribing a d-simplex $\sigma$ whose vertices are rational points is mapped by $\phi$ to a rational point.

Proof. $\phi(S)=\bigcap_{p \in \mathcal{P}_{\sigma}} \phi(p)^{*}$. Each $\phi(p)^{*}$ is a rational hyperplane of $\mathbb{E}^{d+1}$, so, the intersection is rational.

Let us remark that a sphere $S \subset \mathbb{E}^{d}$ has rational Euclidean circumcenter and squared radius iff its associated point $\phi(S)$ in the space of spheres has rational coordinates.

The equation of cone $C$ in $\mathbb{E}^{d+1}$ is given as follows

$$
(x, \chi) \in C \Longleftrightarrow\|x\|^{2}-\left(\frac{1+\chi}{2}\right)^{2}<0 .
$$

Proposition 10. The evaluation of the is_hyperbolic predicate, which tests whether a k-simplex of $D T_{\mathbb{E}}(\mathcal{P})$ belongs to $D T_{\mathbb{H}}(\mathcal{P})$, can be performed using rational computations only.

Proof. Let $\sigma$ be a $k$-simplex in $\operatorname{DT}_{\mathbb{E}}(\mathcal{P})$ and $\left\{\tau_{i}, 0 \leq\right.$ $i<m\}$ be the collection of its incident $(k+1)$-simplices in $\operatorname{DT}_{\mathbb{E}}(\mathcal{P})$. Then the corresponding $(d-k)$-face $u_{\sigma}$ of $U_{\mathcal{P}}$ is given by Equation (2) (see Section 2). Thus, the construction of $u_{\sigma}$ involves rational computations only. As shown by Lemma 8 , the central projection $\psi_{\pi_{\infty}}\left(u_{\sigma}\right)$ of $u_{\sigma}$ onto $\pi_{\infty}$ is rational as well.
The projection $n_{\sigma}$ of the origin $O_{\pi_{\infty}}$ onto the $(d-k)$ flat $\psi_{\pi_{\infty}}\left(\bigcap_{p \in \mathcal{P}_{\sigma}} \phi(p)^{*}\right)$ supporting $\psi_{\pi_{\infty}}\left(u_{\sigma}\right)$, as defined in Section 4.2 , is given by the intersection of the normal to this $(d-k)$-flat passing through $O_{\pi_{\infty}}$ and itself, thus it is rational.

It remains to say that the test whether $n_{\sigma}$ lies in the convex polyhedron given by Equation (2) boils down to orientation tests, and inclusion in $\mathcal{B}_{\pi_{\infty}}$ reduces to comparing its square distance to $O_{\pi_{\infty}}$ with 1 .

Altogether, Sections 5.1 and 5.2 show that the combinatorial structure of $\mathrm{DT}_{\mathbb{H}}(\mathcal{P})$ can be computed using only rational computations.

\subsection{Computing the embedding of $\mathbf{D T}_{\mathbb{H}}(\mathcal{P})$ in the Poincaré ball}

Let us now focus on the geometric embedding of $\mathrm{DT}_{\mathbb{H}}(\mathcal{P})$.

As already mentioned, a hyperplane (i.e., a $(d-1)$-flat) in $\mathbb{H}^{d}$ is a portion of a Euclidean $d$-sphere. We say that the hyperplane is rational if the corresponding Euclidean sphere as a rational equation, i.e., if its Euclidean center has rational coordinates and its squared Euclidean radius is rational, or equivalently, if it is mapped by $\phi$ to a rational point.

A $k$-flat in $\mathbb{H}^{d}$, for $k>0$, is given by the intersection of $d-k$ hyperplanes. We say that the $k$-flat is rational if all these hyperplanes are rational. Then we inductively define a $k$-simplex to be rational if its supporting $k$-flat is rational and if its faces of dimensions $0, \ldots, k-1$ are rational.

Proposition 11. In the Poincaré ball model, the geometric embedding of the hyperplane supporting any facet of $D T_{\mathbb{H}}(\mathcal{P})$ is rational.

Proof. A facet of $\mathrm{DT}_{\mathbb{H}}(\mathcal{P})$ is a $(d-1)$-simplex supported by the hyperplane containing its vertices $p_{0}, p_{1}, \ldots, p_{d-1}$. This hyperplane is embedded in the Poincaré ball model as the Euclidean sphere $S$ that passes through the $p_{i}$ 's and that is orthogonal to $\mathcal{H}_{\infty}$, i.e.,

$$
\phi(S)=\bigcap_{0 \leq i<d-1} \phi\left(p_{i}\right)^{*} \bigcap \pi_{\infty} .
$$

It is the intersection of hyperplanes of $\mathbb{E}^{d+1}$, which are all rational.

Corollary 12. The embedding of $D T_{\mathbb{H}}(\mathcal{P})$ is rational.

Proof. By hypothesis on $\mathcal{P}$, each vertex of $\mathrm{DT}_{\mathbb{H}}(\mathcal{P})$ has rational coordinates. Each edge (1-simplex) of $\operatorname{DT}_{\mathbb{H}}(\mathcal{P})$ is supported by the intersection of $d-1$ hyperplanes, and its endpoints are rational vertices. The edge will be rational iff all hyperplanes are rational. In the same way, a $k$-simplex $\operatorname{DT}_{\mathbb{H}}(\mathcal{P})$, for any $k>0$, will be rational iff all $d-k$ hyperplanes defining its supporting flat are rational. All considered hyperplanes are rational from Proposition 11, which concludes the proof.

\subsection{Computing the embedding of $\operatorname{VD}_{\mathbb{H}}(\mathcal{P})$ in the Poincaré ball}

Let us move on to the geometrical embedding of the hyperbolic Voronoi diagram $\operatorname{VD}_{\mathbb{H}}(\mathcal{P})$.

Proposition 13. The bisector of two points of $\mathcal{P}$ is a hyperbolic hyperplane whose equation in $\mathbb{E}^{d}$ is rational. 


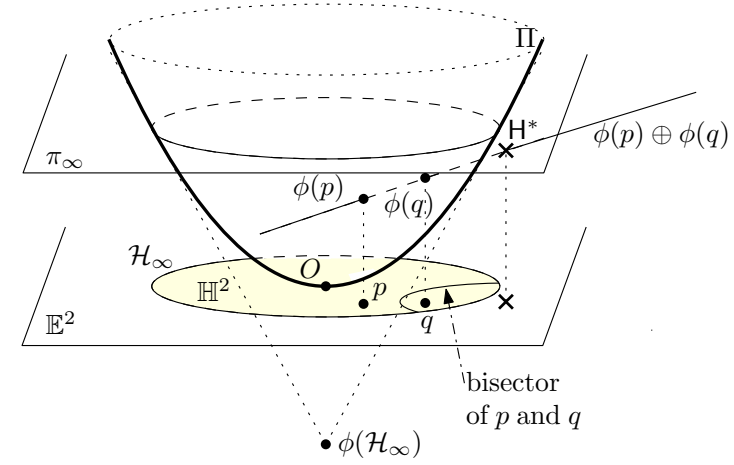

Figure 7: $\mathrm{H}^{*}$ is the image by $\phi$ of the bisector of $p$ and $q$ in $\mathbb{H}^{2}$.

Proof. Let $p$ and $q$ be two points of $\mathcal{P}$. In the space of spheres, We are going to construct their hyperbolic bisector as the locus of hyperbolic centers of spheres passing through both of them.

The intersection $\phi(p)^{*} \cap \phi(q)^{*}$ is the $(d-1)$-flat in the space of spheres $\mathbb{E}^{d+1}$ that represent all spheres of $\mathbb{E}^{d}$ passing through $p$ and $q$. By the construction explained earlier for hyperbolic centers, $\psi_{\Pi}\left(\phi(p)^{*} \cap \phi(q)^{*}\right)$ is the image by $\phi$ of the set of all centers of these spheres. By definition of $\psi_{\Pi}$, it is the intersection of the hyperplane $\mathbf{H}=\left(\phi(p)^{*} \cap \phi(q)^{*}\right) \oplus$ $\phi\left(\mathcal{H}_{\infty}\right)$ with $\Pi$.

The polar of this hyperplane is the point $\mathbf{H}^{*}=(\phi(p) \oplus$ $\phi(q)) \cap \pi_{\infty}$, using Equation (1). Consequently, $\mathrm{H} \cap \Pi$ is the image by $\phi$ of a sphere in $\mathbb{E}^{d}$, which is the set of centers of spheres through $p$ and $q$. All steps in the construction involve only rational computations. Figure 7 illustrates it for $d=2$.

Since a $k$-face in $\operatorname{VD}_{\mathbb{H}}(\mathcal{P})$ is the bisector of $k$ points of $\mathcal{P}$, it is the intersection of $k-1$ rational hyperplanes, we deduce that

Corollary 14. The bisector of $k$ points, for $2 \leq k \leq d$, is a hyperbolic $(d-k+1)$-flat whose equation is rational.

For $k=d+1$, this means that the equation of a Voronoi vertex, seen as a Euclidean 0-sphere, is rational. However:

Proposition 15. The coordinates of a hyperbolic Voronoi vertex are algebraic numbers of degree 2.

Proof. There are at least two ways of seeing this result. One is to consider a hyperbolic Voronoi vertex as the intersection of $d$ hyperplanes in $\mathbb{H}^{d}$, i.e., $d$ Euclidean spheres $S_{0}, \ldots, S_{d-1}$. This is also the intersection of $S_{0}$ with the $d-1$ radical hyperplanes of $S_{0}$ and $S_{i}, 1<i<d$. Each radical hyperplane is rational, since its equation is obtained as the difference of the equations of the two corresponding spheres. The Voronoi vertex is the intersection point between a sphere and a line that lies in $\mathcal{B}$, which shows the result.

A direct construction of the hyperbolic center $x$ of a sphere of Euclidean center $c$ and radius $r$, using $\psi_{\Pi}$ (see Figures 2 and 8), shows that $\|x\|$ is the smallest solution of $\|x\|=$ $\frac{1+\|x\|^{2}}{1+c^{2}-r^{2}} \cdot\|c\|$, then, $x=\frac{1+\|x\|^{2}}{1+c^{2}-r^{2}} \cdot c$.

The intersection of a bisector of dimension $k$ and $\mathcal{H}_{\infty}$ is a rational sphere. For $k=1$, the intersection is a Euclidean

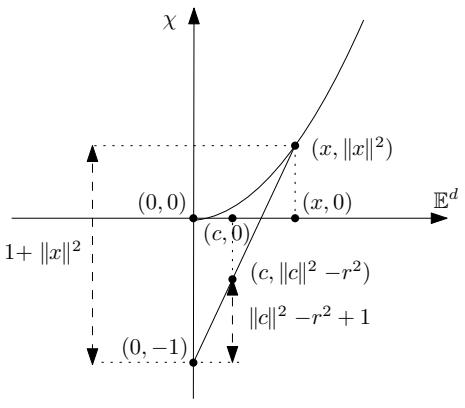

Figure 8: Computation of the hyperbolic center $x$ of a sphere $(c, r)$.

0 -sphere on $\mathcal{H}_{\infty}$; although the equation of this 0 -sphere is rational, the two points of the sphere have coordinates which are algebraic numbers of degree 2. To show this, it suffices to repeat the proof of Proposition 15 considering a point at infinity as the intersection of the sphere $\mathcal{H}_{\infty}$ and $d-1$ Euclidean spheres.

\section{IMPLEMENTATION IN $\mathbb{H}^{2}$}

The construction of the Delaunay complex and the Voronoi diagram in $\mathbb{H}^{2}$ was implemented using CGAL [14, 40, 25] (see [6] for a description). The implementation will soon be submitted for future integration in CGAL. In this section, we detail the computations used, and give some benchmarks.

\subsection{Algebraic and arithmetic aspects}

Let us now detail first the computations of predicates that are needed when implementing the algorithm. Then, the constructions, which are necessary only to compute the geometric embeddings of $\mathrm{DT}_{\mathbb{H}}(\mathcal{P})$ and $\mathrm{VD}_{\mathbb{H}}(\mathcal{P})$, are presented.

\subsubsection{Predicates}

As already mentioned in Section 5.1, we rely on the CGAL package [40] to exactly compute the Euclidean Delaunay triangulation $\mathrm{DT}_{\mathbb{E}}(\mathcal{P})$. The hyperbolic complex $\mathrm{DT}_{\mathbb{H}}(\mathcal{P})$ is then extracted from $\mathrm{DT}_{\mathbb{E}}(\mathcal{P})$ using the predicate is_hyperbolic detailed in Sections 4.2 and 5.2. This predicate is called on Euclidean Delaunay triangles. Non-hyperbolic edges are deduced from non-hyperbolic triangles using the bijection introduced in Corollary 7 .

is_hyperbolic predicate for a triangle. Let $p p^{\prime} p^{\prime \prime}$ be a triangle of $\mathrm{DT}_{\mathbb{E}}(\mathcal{P})$ and $S_{p p^{\prime} p^{\prime \prime}}$ its circumscribing circle. As noticed in Lemma $9, \phi\left(S_{p p^{\prime} p^{\prime \prime}}\right)=\phi(p)^{*} \cap \phi\left(p^{\prime}\right)^{*} \cap \phi\left(p^{\prime \prime}\right)^{*}$.

The equation of $\phi(p)^{*}$ is

$$
\phi(p)^{*}: 2 p_{0} x_{0}+2 p_{1} x_{1}-\chi={p_{0}}^{2}+{p_{1}}^{2}
$$

where $\left(x_{0}, x_{1}, \chi\right)$ is a point of $\mathbb{E}^{3}$.

Solving the linear system $\phi(p)^{*}, \phi\left(p^{\prime}\right)^{*}, \phi\left(p^{\prime \prime}\right)^{*}$, we get

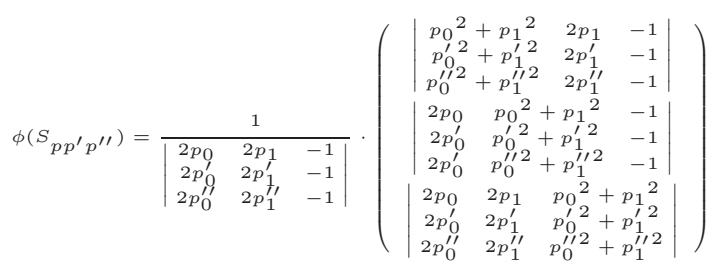



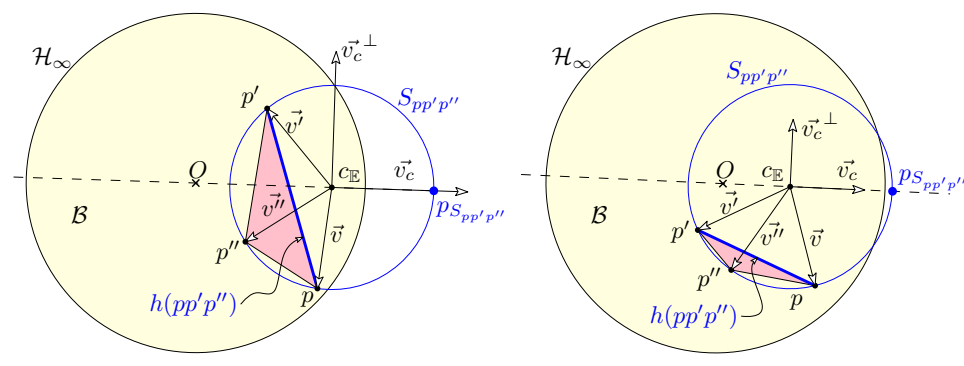

Figure 9: Determining the edge $h\left(p p^{\prime} p^{\prime \prime}\right)$.

Plugging these coordinates in Equation (3) describing the cone $C$ we get that $S_{p p^{\prime} p^{\prime \prime}}$ is hyperbolic if

$\left|\begin{array}{ccc}p_{0}^{2}+p_{1}^{2} & p_{1} & 1 \\ p_{0}^{\prime 2}+p_{1}^{\prime 2} & p_{1}^{\prime} & 1 \\ p_{0}^{\prime \prime 2}+p_{1}^{\prime \prime 2} & p_{1}^{\prime \prime} & 1\end{array}\right|^{2}+\left|\begin{array}{ccc}p_{0} & p_{0}^{2}+p_{1}^{2} & 1 \\ p_{0}^{\prime} & p_{0}^{\prime}+p_{1}^{\prime 2} & 1 \\ p_{0}^{\prime} & p_{0}^{\prime \prime 2}+p_{1}^{\prime \prime 2} & 1\end{array}\right|^{2}-\left|\begin{array}{ccc}p_{0} & p_{1} & p_{0}^{2}+p_{1}^{2}-1 \\ p_{0}^{\prime} & p_{1}^{\prime} & p_{0}^{\prime 2}+p_{1}^{\prime 2}-1 \\ p_{0}^{\prime \prime} & p_{1}^{\prime \prime} & p_{0}^{\prime \prime 2}+p_{1}^{\prime \prime 2}-1\end{array}\right|^{2}<0$

Thus we get the following lemma:

LEMma 16. In $\mathbb{H}^{2}$, the predicate is_hyperbolic applied to a triangle can be evaluated as the sign of a polynomial of degree 8 in the coordinates of its vertices.

is_hyperbolic predicate for an edge. Let $p p^{\prime} p^{\prime \prime}$ be a triangle of $\mathrm{DT}_{\mathbb{E}}(\mathcal{P}) \backslash \mathrm{DT}_{\mathbb{H}}(\mathcal{P})$ with circumscribing circle $S_{p p^{\prime} p^{\prime \prime}}$. The vertices $p, p^{\prime}, p^{\prime \prime}$ are given in counterclockwise order. Corollary 7 and Proposition 2 applied to $\operatorname{DT}_{\mathbb{E}}\left(\left\{p, p^{\prime}, p^{\prime \prime}\right\}\right)$, yield that $h\left(p p^{\prime} p^{\prime \prime}\right)$ is the unique edge in $\operatorname{DT}_{\mathbb{E}}\left(\left\{p, p^{\prime}, p^{\prime \prime}\right\}\right) \backslash$ $\mathrm{DT}_{\mathbb{H}}\left(\left\{p, p^{\prime}, p^{\prime \prime}\right\}\right)$. Let $c_{\mathbb{E}}$ be the Euclidean center of $S_{p p^{\prime} p^{\prime \prime}}$. We define the vectors $\vec{v}=p-c_{\mathbb{E}}, \overrightarrow{v^{\prime}}=p^{\prime}-c_{\mathbb{E}}, \overrightarrow{v^{\prime \prime}}=p^{\prime \prime}-c_{\mathbb{E}}$, which verify $\|\vec{v}\|=\left\|\overrightarrow{v^{\prime}}\right\|=\left\|\overrightarrow{v^{\prime \prime}}\right\|$ and $\vec{v}, \overrightarrow{v^{\prime}}, \overrightarrow{v^{\prime \prime}}$ are in counterclockwise order.

Let us define $\overrightarrow{v_{c}}=\overrightarrow{O c_{\mathbb{E}}}$. The vectors $\vec{v}, \overrightarrow{v^{\prime}}, \overrightarrow{v^{\prime \prime}}$ split the circle $S_{p p^{\prime} p^{\prime \prime}}$ into three (2D) spherical caps. The ray of origin $c_{\mathbb{E}}$ and direction $\overrightarrow{v_{c}}$ intersects $S_{p p^{\prime} p^{\prime \prime}}$ in $p_{S_{p p^{\prime} p^{\prime \prime}} \text {, which }}$ lies in one of these three caps, and outside $\mathcal{B}^{p p^{\prime}}$. The cap that contains $p_{S_{p p^{\prime} p^{\prime \prime}}}$ is supported by $h\left(p p^{\prime} p^{\prime \prime}\right)$ and can be uniquely determined by the two vectors from $\vec{v}, \overrightarrow{v^{\prime}}, \overrightarrow{v^{\prime \prime}}$ that define a wedge in counterclockwise order containing $\overrightarrow{v_{c}}$ (see Figure 9).

The computation of $h\left(p p^{\prime} p^{\prime \prime}\right)$ boils down to computing Counterclockwise $\left(\vec{v}, \overrightarrow{v_{c}}, \overrightarrow{v^{\prime}}\right), \quad$ Counterclockwise $\left(\overrightarrow{v^{\prime}}, \overrightarrow{v_{c}}, \overrightarrow{v^{\prime \prime}}\right)$, and Counterclockwise $\left(\overrightarrow{v^{\prime \prime}}, \overrightarrow{v_{c}}, \vec{v}\right)$, where Counterclockwise $\left(\vec{w}, \overrightarrow{v_{c}}, \overrightarrow{w^{\prime}}\right)$ tests whether the three vectors $\vec{w}, \overrightarrow{v_{c}}$, and $\overrightarrow{w^{\prime}}$ are in counterclockwise order. ${ }^{2}$

It remains to detail the computation of Counterclockwise $\left(\vec{w}, \overrightarrow{v_{c}}, \overrightarrow{w^{\prime}}\right)$, where $\|w\|=\left\|w^{\prime}\right\|$ (notice that $\left\|\overrightarrow{v_{c}}\right\|$ has no reason to be equal to $\|\vec{w}\|)$. Let $\overrightarrow{v_{c}}$ be the vector obtained by rotating $\overrightarrow{v_{c}}$ by $\frac{\pi}{2}$. If $\operatorname{sign}\left(\vec{w} \cdot{\overrightarrow{v_{c}}}^{\perp}\right)=-\operatorname{sign}\left(\overrightarrow{w^{\prime}} \cdot{\overrightarrow{v_{c}}}^{\perp}\right)=-1$ (which holds e.g. for $\vec{v}$ and $\overrightarrow{v^{\prime}}$ in Figure 9-left) then Counterclockwise $\left(\vec{w}, \overrightarrow{v_{c}}, \overrightarrow{w^{\prime}}\right)$ is true. If $\operatorname{sign}\left(\vec{w} \cdot \overrightarrow{v_{c}}{ }^{\perp}\right)=\operatorname{sign}\left(\overrightarrow{w^{\prime}} \cdot{\overrightarrow{v_{c}}}^{\perp}\right)=$ -1 (see Figure 9-right) then Counterclockwise $\left(\vec{w}, \overrightarrow{v_{c}}, \overrightarrow{w^{\prime}}\right)$ is equal to Orientation $\left(\vec{w}, \overrightarrow{v_{c}}, \overrightarrow{w^{\prime}}\right)$. Other cases are symmetric.

From Equation (5),

$c_{\mathbb{E}}=\frac{1}{\left|\begin{array}{ccc}2 p_{0} & 2 p_{1} & -1 \\ 2 p_{0}^{\prime} & 2 p_{1}^{\prime} & -1 \\ 2 p_{0}^{\prime \prime} & 2 p_{1}^{\prime \prime} & -1\end{array}\right|}\left(\left|\begin{array}{ccc}p_{0}^{2}+p_{1}^{2} & 2 p_{1} & -1 \\ p_{0}^{\prime} 2+p_{1}^{\prime} 2 & 2 p_{1}^{\prime} & -1 \\ p_{0}^{\prime \prime 2}+p_{1}^{\prime \prime 2} & 2 p_{1}^{\prime \prime} & -1\end{array}\right|,\left|\begin{array}{ccc}2 p_{0} & p_{0}^{2}+p_{1}^{2} & -1 \\ 2 p_{0}^{\prime} & p_{0}^{\prime}+p_{1}^{\prime 2} & -1 \\ 2 p_{0}^{\prime} & p_{0}^{\prime \prime 2}+p_{1}^{\prime \prime 2} & -1\end{array}\right|\right)$

${ }^{2}$ Degeneracies in the Counterclockwise test cannot occur since they would correspond either to equality of two points of $\left\{p, p^{\prime}, p^{\prime \prime}\right\} \subset \mathcal{B}$ or equality of one of these points to $p_{S_{p p^{\prime} p^{\prime \prime}}} \notin \mathcal{B}$.
So, the coordinates of $\vec{v}, \overrightarrow{v^{\prime}}, \overrightarrow{v^{\prime \prime}}$, and $\overrightarrow{v_{c}}$ are rational fractions with numerators of degree 3 and a common denominator of degree 2. Thus the signs of the above scalar products and Orientation tests boil down to signs of polynomials of degree 6 , and we get the following lemma:

LEMMA 17. The non hyperbolic edge associated to a non hyperbolic triangle by the map $h$ can be determined by the evaluation of the signs of polynomials of degree 6 in the coordinates of its vertices.

Exact evaluation. We have seen that all predicate evaluations boil down to computing signs of polynomials. As for the CGAL Euclidean Delaunay triangulations (section 5.1), this can be done in a fast and exact way using filtered exact computations, providing an efficient and fully robust implementation.

\subsubsection{Constructions}

To draw the Delaunay triangulation we need to construct the hyperbolic line through two points, and to draw the Voronoi diagram we need to construct the hyperbolic bisector of two points and the hyperbolic center of the circle through three points.

Hyperbolic line construction. The hyperbolic line through $p$ and $p^{\prime}$ is supported by the Euclidean circle $S$ such that:

$$
\begin{aligned}
& \phi(S)=\phi(p)^{*} \cap \phi\left(p^{\prime}\right)^{*} \cap \pi_{\infty}= \\
& \left(-\frac{\left|\begin{array}{ll}
p_{1} & p_{0}^{2}+p_{1}^{2}-1 \\
p_{1}^{\prime} & p_{0}^{\prime}+p_{1}^{\prime}-1
\end{array}\right|}{p_{0}^{\prime} p_{1}-p_{0} p_{1}^{\prime}}, \frac{\left|\begin{array}{ll}
p_{0} & p_{0}^{2}+p_{1}^{2}-1 \\
p_{0}^{\prime} & p_{0}^{\prime}+p_{1}^{\prime 2}-1
\end{array}\right|}{p_{0}^{\prime} p_{1}-p_{0} p_{1}^{\prime}}, 1\right) .
\end{aligned}
$$

Hyperbolic bisector construction. As noticed in the proof of Lemma 13, the hyperbolic bisector of $p$ and $p^{\prime}$ is suported by the Euclidean circle $S$ such that $\phi(S)=$ line $\left(\phi(p), \phi\left(p^{\prime}\right)\right) \cap \pi_{\infty}$.

$$
\begin{aligned}
\phi(S)= & \frac{p_{0}^{\prime 2}+p_{1}^{\prime 2}}{2\left(p_{0}^{2}+p_{1}^{2}\right)\left(p_{0}^{\prime 2}+p_{1}^{\prime 2}\right)}\left(p_{0}, p_{1}, p_{0}{ }^{2}+p_{1}{ }^{2}\right) \\
& +\frac{p_{0}{ }^{2}+p_{1}{ }^{2}}{2\left(p_{0}{ }^{2}+p_{1}{ }^{2}\right)\left(p_{0}^{\prime 2}+p_{1}^{\prime 2}\right)}\left(p_{0}^{\prime}, p_{1}^{\prime}, p_{0}^{\prime 2}+p_{1}^{\prime 2}\right)
\end{aligned}
$$

Hyperbolic circumcenter construction. As observed in the proof of Proposition 15 the hyperbolic center $x$ of the triangle $p p^{\prime} p^{\prime \prime}$ can be computed as $\psi_{\Pi}\left(\phi(p)^{*} \cap \phi\left(p^{\prime}\right)^{*} \cap \phi\left(p^{\prime \prime}\right)^{*}\right)$.

Let $\frac{1}{\delta}(\alpha, \beta, \gamma)$ be $\left.\phi(p)^{*} \cap \phi\left(p^{\prime}\right)^{*} \cap \phi\left(p^{\prime \prime}\right)^{*}\right)$, we have already computed in Equation 5

$$
\begin{gathered}
\delta=\left|\begin{array}{ccc}
2 p_{0} & 2 p_{1} & -1 \\
2 p_{0}^{\prime} & 2 p_{1}^{\prime} & -1 \\
2 p_{0}^{\prime \prime} & 2 p_{1}^{\prime \prime} & -1
\end{array}\right|, \quad \alpha=\left|\begin{array}{ccc}
p_{0}^{2}+p_{1}^{2} & 2 p_{1} & -1 \\
p_{0}^{\prime 2}+p_{1}^{\prime 2} & 2 p_{1}^{\prime} & -1 \\
p_{0}^{\prime \prime 2}+p_{1}^{\prime \prime 2} & 2 p_{1}^{\prime \prime} & -1
\end{array}\right|, \\
\beta=\left|\begin{array}{ccc}
2 p_{0} & p_{0}^{2}+p_{1}^{2} & -1 \\
2 p_{0}^{\prime} & p_{0}^{\prime 2}+p_{1}^{\prime 2} & -1 \\
2 p_{0}^{\prime} & p_{0}^{\prime \prime 2}+p_{1}^{\prime \prime 2} & -1
\end{array}\right|, \quad \gamma=\left|\begin{array}{ccc}
2 p_{0} & 2 p_{1} & p_{0}^{2}+p_{1}^{2} \\
2 p_{0}^{\prime} & 2 p_{1}^{\prime} & p_{0}^{\prime 2}+p_{1}^{\prime 2} \\
2 p_{0}^{\prime \prime} & 2 p_{1}^{\prime \prime} & p_{0}^{\prime \prime 2}+p_{1}^{\prime \prime 2}
\end{array}\right|
\end{gathered}
$$

then let $\lambda$ be the smallest solution of equation $\lambda^{2}+$ $\frac{\gamma-\delta}{\sqrt{\alpha^{2}+\beta^{2}}} \lambda-1=0$, that is

$$
\lambda=\frac{\delta-\gamma-\sqrt{(\gamma-\delta)^{2}-4 \alpha^{2}-4 \beta^{2}}}{\sqrt{4 \alpha^{2}+4 \beta^{2}}}
$$

and the hyperbolic center is $x=\frac{1+\lambda^{2}}{\delta(1+\gamma)}(\alpha, \beta)$. 


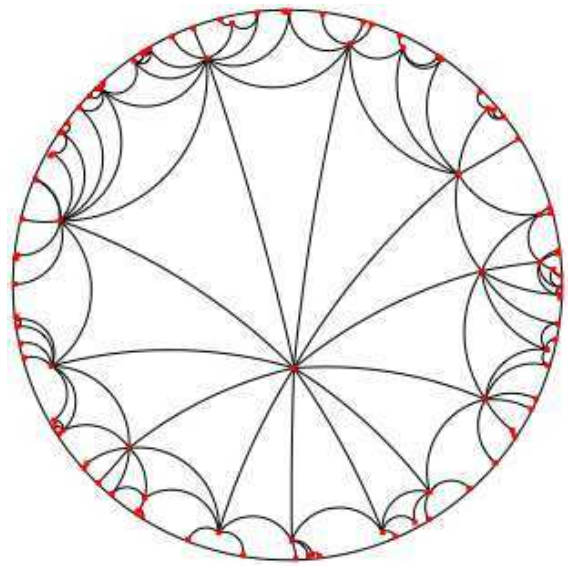

Figure 10: 100 uniformly distributed points according to the hyperbolic metric in an open ball in $\mathbb{H}^{2}$.

Evaluation. CGAL provides us with number type Sqrt_extension for exact computations on algebraic numbers of degree 2 [26], allowing us to construct in an exact way the hyperbolic center of a circle as well as hyperbolic bisector between two input points. These constructions are rounded only when displaying the hyperbolic Voronoi diagram.

\subsection{Experimental results}

Experiments are run on point sets $\mathcal{P}$ that are uniformly distributed (up to rounding errors when generating them) according to the hyperbolic metric in open balls in $\mathcal{B}$ (Figure 10 shows an example with 100 points). Each open ball is centered at the origin of $\mathcal{B}$ with Euclidean radius $1-\epsilon$. The point sets in the open balls are denoted by small_sph for $\epsilon=10^{-3}$ and by big_sph for $\epsilon=10^{-7}$.

We insert all points in $\mathcal{P}$ at once, and we measure the running times of the computations of $\mathrm{DT}_{\mathbb{E}}(\mathcal{P})$ with the CGAL implementation [40] and of $\mathrm{DT}_{\mathbb{H}}(\mathcal{P})$ with our implementation of the algorithm of Section 4.1.2. This allows us to measure the overhead due the static extraction of the Delaunay complex in $\mathbb{H}^{2}$.

The table for the results is organized as follows. The first column gives the number of input points. The second and the third column give running times in seconds for the computations of $\mathrm{DT}_{\mathbb{E}}(\mathcal{P})$ and $\mathrm{DT}_{\mathbb{H}}(\mathcal{P})$, respectively. The fourth column shows the overhead factor of the computation of $\mathrm{DT}_{\mathbb{H}}(\mathcal{P})$ compared to $\mathrm{DT}_{\mathbb{E}}(\mathcal{P})$. Experiments are conducted on a MacBookPro 2.6 GHz running CGAL 4.0 in release mode, using GCC 4.2. Running times are averaged on 10 trials. For both $\operatorname{DT}_{\mathbb{E}}(\mathcal{P})$ and $\operatorname{DT}_{\mathbb{H}}(\mathcal{P})$, we use CGAL: :Exact_predicates_inexact_constructions_kernel, which provides filtered exact geometric predicates.

\begin{tabular}{l|l|l|l} 
small_sph & $\mathbb{E}^{2}$ & $\mathbb{H}^{2}$ & factor \\
\hline $10^{4}$ & 0.00805 & 0.0108 & 1.34 \\
$10^{5}$ & 0.0867 & 0.0982 & 1.13 \\
$10^{6}$ & 0.890 & 0.935 & 1.05 \\
$10^{7}$ & 9.61 & 9.80 & 1.02 \\
big_sph & $\mathbb{E}^{2}$ & $\mathbb{H}^{2}$ & factor \\
\hline $10^{4}$ & 0.00745 & 0.0181 & 2.43 \\
$10^{5}$ & 0.0833 & 0.177 & 2.12 \\
$10^{6}$ & 0.898 & 1.07 & 1.18 \\
$10^{7}$ & 10.1 & 10.4 & 1.02
\end{tabular}

We observe that the overhead of the extraction decreases with the size of the input point set, and becomes very small $(2 \%)$ for large point sets. The reason is that the graph of faces that is traversed by the extraction scheme grows in a slower way than the whole graph of the Euclidean triangulation. Faces examined during the extraction are in some sense "close" to the convex hull of the point set.

As could be expected, the overhead for small_sph is better than the overhead obtained on big_sph for larger point sets, since a small fraction of Euclidean Delaunay circles intersect $\mathcal{H}_{\infty}$.

We also observed that the ratio (\# edges in $D T_{\mathbb{E}}(\mathcal{P})$ ) / (\# edges in $D T_{\mathbb{H}}(\mathcal{P})$ ) quickly decreases with the number of vertices. For instance, on big_sph with $10^{7}$ points, this ratio is 1.006 .

More experiments can be found in [6].

\section{CONCLUSION AND OPEN PROBLEMS}

We proposed an algorithm to compute hyperbolic Voronoi diagrams and Delaunay complexes, as well as a complete and efficient implementation, to be submitted to CGAL.

We are pursuing research on periodic hyperbolic Delaunay triangulations, in the flavor of what we had proposed for the Euclidean case $[12,13,7]$. This is motivated by applications in various fields such as geometry processing [32].

Our implementation has already been used to compute periodic meshes of the hyperbolic plane [33] to answer a question raised by colleagues in neuro mathematics [15].

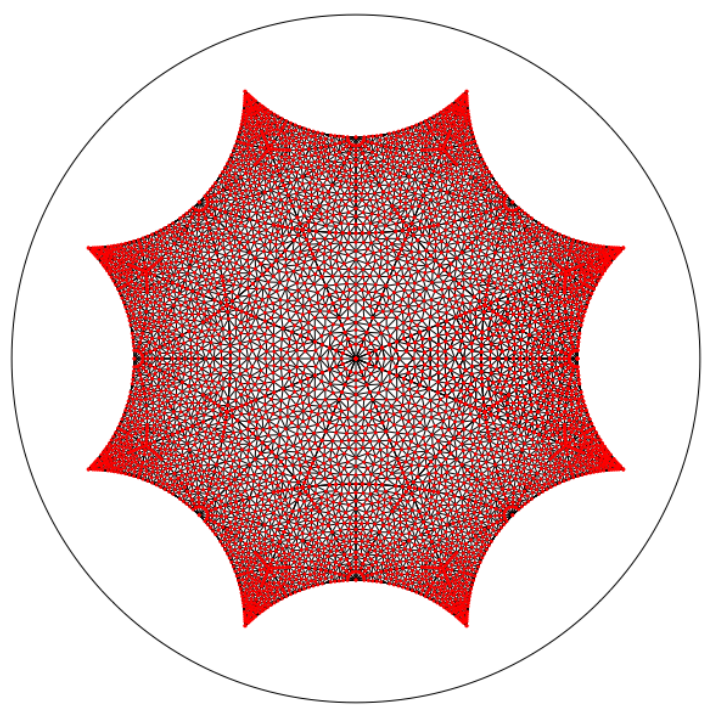

Figure 11: Periodic mesh of the hyperbolic plane respecting the symmetries of the octagon. 


\section{REFERENCES}

[1] F. Aurenhammer. Voronoi diagrams: A survey of a fundamental geometric data structure. ACM Comput. Surv., 23:345-405, 1991. doi:10.1145/116873.116880.

[2] F. Aurenhammer and R. Klein. Voronoi diagrams. In J.-R. Sack and J. Urrutia, editors, Handbook of Computational Geometry, 201-290. Elsevier, 2000. ftp://ftp.cis. upenn.edu/pub/cis610/public_htm1/ak-vd-00.ps.

[3] A. F. Beardon. The Geometry of Discrete Groups. Graduate Texts in Math. Springer-Verlag, 1983.

[4] M. Berger. Geometry. Springer-Verlag, 1987.

[5] M. Bern and D. Eppstein. Optimal Möbius transformations for information visualization and meshing. In 7th Workshop on Algorithms and Data Structures, volume 2125 of LNCS, 2001. http://arxiv.org/abs/cs.cG/0101006.

[6] M. Bogdanov, O. Devillers, and M. Teillaud. Hyperbolic Delaunay complexes and Voronoi diagrams made practical. Research Report 8146, INRIA, 2012. http://hal.inria.fr/hal-00756522.

[7] M. Bogdanov, M. Teillaud, and G. Vegter. Covering spaces and Delaunay triangulations of the $2 \mathrm{~d}$ flat torus. In Abstracts 28th European Workshop on Computational Geometry, 2012. ftp://ftp-sop.inria.fr/geometrica/teillaud/eurocg12.pdf .

[8] J.-D. Boissonnat, A. Cérézo, O. Devillers, and M. Teillaud. Output-sensitive construction of the Delaunay triangulation of points lying in two planes. Internat. J. Comput. Geom. Appl., 6:1-14, 1996. doi:10.1142/S0218195996000022.

[9] J.-D. Boissonnat and M. Yvinec. Algorithmic Geometry. Cambridge Univ. Press, 1998.

[10] A. Bowyer. Computing Dirichlet tesselations. Comput. J., 24:162-166, 1981. doi:10.1093/comjnl/24.2.162.

[11] J. Cannon, W. Floyd, R. Kenyon, and W. Parry. Hyperbolic geometry. Flavors of geometry, 31:59-115, 1997.

[12] M. Caroli and M. Teillaud. Computing 3d periodic triangulations. In ESA, volume 5757 of $L N C S, 37-48$, 2009. http:// /ww . springerlink. com/content/55415144316j214g/fulltext.pdf.

[13] M. Caroli and M. Teillaud. Delaunay triangulations of point sets in closed Euclidean d-manifolds. In Symposium on Computational Geometry, 274-282, 2011. doi:10.1145/1998196.1998236.

[14] CGal, Computational Geometry Algorithms Library. http://www. cgal.org.

[15] P. Chossat, G. Faye, and O. Faugeras. Bifurcation of hyperbolic planforms. Journal of Nonlinear Science, 21:465-498, 2011. doi:10.1007/s00332-010-9089-3.

[16] M. de Berg, M. van Kreveld, M. Overmars, and O. Schwarzkopf. Computational Geometry: Algorithms and Applications. Springer-Verlag, 1997. http:///ww .cs.uu.nz/geobook/.

[17] O. Devillers. The Delaunay hierarchy. Internat. J. Found. Comput. Sci., 13:163-180, 2002. doi: 10.1142/S0129054102001035.

[18] O. Devillers, S. Meiser, and M. Teillaud. The space of spheres, a geometric tool to unify duality results on Voronoi diagrams. In Canad. Conf. Computational Geometry, 263-268, 1992. INRIA RR 1620.

http://hal.inria.fr/inria-00074941.
[19] O. Devillers and S. Pion. Efficient exact geometric predicates for Delaunay triangulations. In Workshop on Algorithm Engineering and Experimentation, 37-44, 2003. http://hal.inria.fr/inria-00344517/.

[20] O. Devillers, S. Pion, and M. Teillaud. Walking in a triangulation. Internat. J. Found. Comput. Sci., 13:181-199, 2002. doi:10.1142/\$0129054102001047.

[21] O. Devillers and M. Teillaud. Perturbations for Delaunay and weighted Delaunay 3D triangulations. Computational Geometry: Theory and Applications, 44:160-168, 2011. doi:10.1016/j.comgeo.2010.09.010.

[22] H. Edelsbrunner and R. Seidel. Voronoi diagrams and arrangements. Discrete Comput. Geom., 1:25-44, 1986. doi:10.1007/BF02187681.

[23] D. Eppstein. Hyperbolic geometry, Möbius transformations, and geometric optimization, 2003. Invited talk at MSRI Introductory Workshop on Discrete and Computational Geometry. http://www.msri.org/realvideo//n/msri/2003/introdcgeom/eppstein/2/meta/aux/eppstein2.pdf .

[24] D. Eppstein and M. Goodrich. Succinct greedy graph drawing in the hyperbolic plane. In International Symposium on Graph Drawing, volume 5417 of LNCS, 14-25, 2008. http://arxiv. org/abs/0806.0341.

[25] E. Fogel and M. Teillaud. Generic programming and the CGAL library. In J.-D. Boissonnat and M. Teillaud, editors, Effective Comp. Geom. for Curves and Surfaces. Springer-Verlag, Mathematics and Visualization, 2006.

[26] M. Hemmer, S. Hert, L. Kettner, S. Pion, and S. Schirra. Number types. In CGAL Manual. CGAL Ed. Board, 4.0 edition, 2012.

http://wwu.cgal.org/Manual/1atest/doc_htm//cgal_manual/packages.html\#pkg: NumberTypes

[27] M. Hurdal and K. Stephenson. Cortical cartography using the discrete conformal approach of circle packings. Neuroimage, 23:119-128, 2004.

[28] M. Jin, J. Kim, F. Luo, and X. Gu. Discrete surface Ricci flow. IEEE Trans. Vis. Comput. Graph., 14:1030-1043, 2008.

[29] T. Munzner. Exploring large graphs in 3D hyperbolic space. IEEE Computer Graphics \&5 App., 18:18-23, 1998.

[30] F. Nielsen and R. Nock. Hyperbolic Voronoi diagrams made easy. In Int. Conf. on Computational Science and its App., 74-80. IEEE, 2010. doi:10.1109/ICCSA.2010.37.

[31] K. Onishi and N. Takayama. Construction of Voronoi diagrams on the upper half-plane. IEICE Trans. Fundamentals, E79-A:533-539, 1996.

[32] G. Ron, M. Jin, and X. Guo. Hyperbolic centroidal Voronoi tessellation. In ACM Symp. on Solid and Physical Modeling, 117-126, 2010. doi:10.1145/1839778.1839795.

[33] M. Schmitt and M. Teillaud. Meshing the hyperbolic octagon. Research Report 8179, INRIA, 2012. http://hal.inria.fr/hal-00764965.

[34] T. Tanuma, H. Imai, and S. Moriyama. Revisiting hyperbolic Voronoi diagrams from theoretical, applied and generalized viewpoints. In Internat. Symp. on Voronoi Diagrams in Science and Engineering, 23-32. IEEE Computer Society, 2010. doi:10.1109/ISVD.2010.13.

[35] W. Thurston. Three dimensional manifolds, Kleinian groups, and hyperbolic geometry. Bull. Amer. Math. 
Soc., 6:357-381, 1982. http://wwu. ams. org/mathscinet-getitem?mr=648524.

[36] W. Thurston. The geometry and topology of three-manifolds, 2002. http://www.msri.org/publications/books/gt3m/.

[37] D. Watson. Computing the $n$-dimensional Delaunay tesselation with applications to Voronoi polytopes. Comput. J., 24:167-172, 1981. doi:10.1093/comjn1/24.2.167.

[38] P. Wilson. Curved Spaces. Cambridge Univ. Press, 2008.

[39] C. Yap and T. Dubé. The exact computation paradigm. In D. Du and F. Hwang, editors, Computing in Euclidean Geometry, volume 4 of Lecture Notes Series on Computing, 452-492. World Scientific, 2nd edition, 1995. http://www.cs.nyu.edu/-exact/doc/paradigm.ps.gz.

[40] M. Yvinec. 2D triangulations. In CGAL Manual. CGAL Ed. Board, 4.0 edition, 2012. http ///www. cgal. org/Manual/1atest/doc_htm1/cgal_manual/packages. htm1\#pkg:Triangulation2.

[41] W. Zeng, R. Sarkar, F. Luo, X. Gu, and J. Gao. Resilient routing for sensor networks using hyperbolic embedding of universal covering space. In Conference on Information communications, INFOCOM'10, 1694-1702. IEEE Press, 2010. 